\title{
An outbreak of multidrug-resistant Serratia marcescens: The importance of continuous monitoring of nosocomial infections
}

\author{
Maida Šiširak ${ }^{1}$, Mirsada Hukić ${ }^{1,2}$ \\ ${ }^{1}$ Clinical Center University of Sarajevo \\ Institute for Clinical microbiology \\ Clinical Center University of Sarajevo \\ Sarajevo, Bosnia and Herzegovina \\ ${ }^{2}$ Department of Medical Sciences \\ of the Academy of Sciences and Arts \\ of Bosnia and Herzegovina, Sarajevo \\ Bosnia and Herzegovina \\ Corresponding author: \\ Maida Šiširak \\ Institute for Clinical Microbiology \\ Clinical Center University of Sarajevo \\ Bolnička 25, 71000 Sarajevo, \\ Bosnia and Herzegovina \\ maidasisirak@yahoo.com \\ Tel.: + 38733226960 \\ Fax.: + 38733226960
}

Received: 11 March 2013

Accepted: 10 May 2013

Copyright (C) 2013 by Academy of Sciences and Arts of Bosnia and Herzegovina. E-mail for permission to publish: amabih@anubih.ba
Objectives. Serratia marcescens is a well-established as a nosocomial pathogen, resulting in considerable morbidity and mortality in immunocompromised patients. The aim of this study was to investigate an outbreak of Serratia marcescens at the Orthopaedic Clinic of the Clinical Center University of Sarajevo. Methods. A total of 96 strains from 79 patients were isolated. The isolates were identified by conventional methods. Susceptibility testing was performed by the discdiffusion method following CLSI guidelines. Results were confirmed by VITEC-2 Compact. Results. From January to December 2010, 96 strains from 79 patients were isolated at the Orthopaedic Clinic of the Clinical Center, University of Sarajevo.The strains were isolated from wound swabs, blood cultures and cerebrospinal fluid. The strains were identifed using current phenotypic methods as Serratia marcescens with identical biochemical characteristics and antibiotic susceptibility patterns. All strains were susceptible to imipenem, meropenem, amikacin, ciprofloxacin, levofloxacin and piperacillin/tazobactam. The infection control team was alerted and after investigation they discovered the same phenotype of Serratia marcescens in the anaesthetic vials used in procedures. This outbreak was extremely difficult to terminate, even with cohorting of patients, sterilisation of equipment, reinforcement of handwashing and deep-cleaning of facilities. The implementation of new control measures terminated the outbreak in February 2011. Conclusion. Continuous monitoring of nosocomial infections is indispensable. Phenotypic characterization of the isolates is useful for studying the relationship of microbial pathogens. The relationship of one clinical isolate to another during an outbreak is important in motivating the search for a common source or mode of transmission.

Key words: Nosocomial infection, Phenotyping, Serratia marcescens.

\section{Introduction}

Serratia marcescens is an opportunistic pathogen, which is now well established as a nosocomial pathogen, resulting in considerable morbidity and mortality in susceptible patients. Serratia marcescens has been implicated as an aetiological agent in every conceivable kind of infection, including respiratory tract infection, urinary tract infection, septicaemia, meningitis and wound infection $(1,2)$. Patients most at risk are those in intensive care units who are subjected to medical devices, especially central venous 
catheters, and those treated with broadspectrum antimicrobial drugs (2). The first description of nosocomial infection caused by Serratia marcescens was Wheat's report of 11 cases over a 6-month period in 1951 at Stanford University Hospital (3). Infections caused by this organism have been reported with increasing frequency since 1960 (4). Since the emergence of Serratia marcescens as a cause of infections was noticed, many aspects of the pathogenicity and virulence of the organism have been studied, including adherence and hydrophobicity, lipopolisaccharide (LPS) and extracellular products (chitinase, several proteases, nuclease and lipase) (2). Serratia marcescens has expressed the ability to survive and grow under extreme conditions, including in disinfectants, antiseptics and double-distilled water (5-7).

Nosocomial epidemics of infections caused by Serratia marcescens have been described in a variety of clinical settings, and different environmental sources have been identifed as reservoirs. Outbreaks have been traced to multiple sources, including contaminated solutions and disinfectants, intravenous fluids, mechanical respirators, intravenous catheters, ultrasonic nebulizers, fiberoptic bronchoscopes, and hand-to-hand transmission by hospital personnel (4-7).

In addition, Serratia marcescens often demonstrates multiple mechanisms of antibiotic resistance $(2,8-10)$, necessitating the use of antibiotics usually kept in reserve. The antibiotics of choice to treat a variety of infections are $\beta$-lactam agents such as penicillins, cephalosporins, monobactams and carbapenems. The introduction of them into therapy was rapidly followed by reports of resistance $(11,12)$. Microorganisms producing extended-spectrum $\beta$-lactamases (ESBLs) were identifed in the early 1980ies (12). Production of ESBLs is the major mechanism of resistance to oxymino-cephalosporins and aztreonam in Gram-negative bacteria $(12,13)$. Carbapenems are generally the last resort in the treatment of infections caused by Serratia marcescens ESBL, because they are not affected by most $\beta$-lactamases, including ESBLs $(12,13)$.

The subject of this study was the investigation of an outbreak of Serratia marcescens, using phenotypic methods and to emphasize the importance of continuous monitoring of nosocomial infections.

\section{Material and methods}

\section{Patient data}

From January to December 2010, we observed an outbreak of Serratia marcescens involving 79 patients, at the Orthopaedic Clinic of the Clinical Center, University of Sarajevo. Before the onset of infection, all patients had been treated by orthopedic surgeons. Approximately within 48 hours after surgery the symptoms of infections occured.

\section{Bacterial isolates and identification}

A total of 96 isolates of Serratia marcescens were collected from 79 patients involved in the outbreak. The strains were isolated from wound swabs (78), blood cultures (17) and cerebrospinal fluid (1). Culture were made at the Institute of Clinical Microbiology of the University of Sarajevo Clinic Centre. Samples were plated on blood agar and $\mathrm{Mac}$ Conkey agar (Becton Dickinson, New Jersey, United States). Mac Conkey agar was used for selective isolation of Enterobacteriacae. These media are specially designed to distinguish lactose fermenting (pink to red) from non lactose-fermenting colonies (colourless or slightly beige). The plates were incubated overnight at $37^{\circ} \mathrm{C}$ in bacteriological incubators. All isolates were identified by conventional biochemical testing (14) and interpretative criteria established by the Clinical Laboratory Standards Institute (CLSI) guidelines (15). Results were con- 
firmed by VITEC 2 Compact (bioMerieux, Marcy l'Etoile, France). Use of the VITEC 2 Compact with the Gram-negative cards (GN13) validated for use with the instrument identifed the isolates as Serratia marcescens.

\section{Susceptibility testing}

Antimicrobial susceptibility testing was performed by the Kirby-Bauer disc-diffusion method on Mueller-Hinton agar (14), according to CLSI guidelines (15). Bacterial inocula were prepared by suspending the freshly grown bacteria in normal sterile saline adjusted to a $0.5 \mathrm{McF}$ arland standard. Antimicrobial susceptibility was determined for ampicillin, amoxiclilin/clavulanic acid, cefotaxime, ceftazidime, ceftriaxone, cefepime, gentamicin, amikacin, ciprofloxacin, levofloxacin, piperacillin/tazobactam, imipenem and meropenem. Results of antimicrobial susceptibility testing were confirmed by VITEC 2 Compact with AST cards.

\section{Infection control}

In June 2010, it became apparent that an outbreak was occuring and infection control measures were reviewed. Patients known to be infected or colonised were isolated in single rooms, where possible. Extensive environmental microbiological investigations were started. Swabs were taken from numerous surfaces, including walls, floors with their edges and corners, doors and door handles, sinks, ventilators, stethoscopes and other personal medical devices. To address the possibility of human carriage, the hands and cellular phones of some of the healthcare workers were checked. Bottles of saline used for dilutions and one phosphate buffer, multiple-dose vials of anaesthetics, drug bottles and boxes, soaps and disinfectants were swabbed and subcultured. The only positive result for the epidemic strain Serratia marcescens was from a multiple-dose vial of anaesthetics used intravenously during procedures.

\section{Statistical analysis}

The statistical data were analyzed by descriptive statistical values (statistical measures of counting-frequencies, percentages).

\section{Ethical statement}

Ethical principles outlined in the World Medical Association Declaration of Helsinki were applied in this study.

\section{Results}

From January to December 2010, in 79 patients hospitalized in the Orthopedic Clinic of the Clinical Center, University of Sarajevo, Serratia marcescens infections were registered. Before the onset of infection, all patients had been treated by orthopedic surgeons. Approximately within 48 hours after surgery, the symptoms of infection occurred. Laboratory findings showed elevated CRP 74 mg/l (range18-166), ESR 40 $\mathrm{mm} / 1 \mathrm{~h}$ (range 6-88), leukocytosis $12.8 \times 10^{9} / 1$ (range7.9-18.6). Wound swabs, blood cultures and one sample of cerebrospinal fluid from patients were sent to the Institute of Clinical Microbiology and all were positive for Serratia marcescens, with identical biochemical characteristics and antibiotic susceptibility patterns. The gender structure of patients was: 31 (39\%) males and $48(61 \%)$ females. The average age of the patients was 48 years (range from 20 to 76). Most of the patients $(n=52 ; 65 \%)$ were older than 50 years.

A total of 96 samples from 79 patients involved in the outbreak were collected. The strains were isolated from wound swabs (78/96; 81\%), blood cultures $(17 / 96 ; 18 \%)$ 


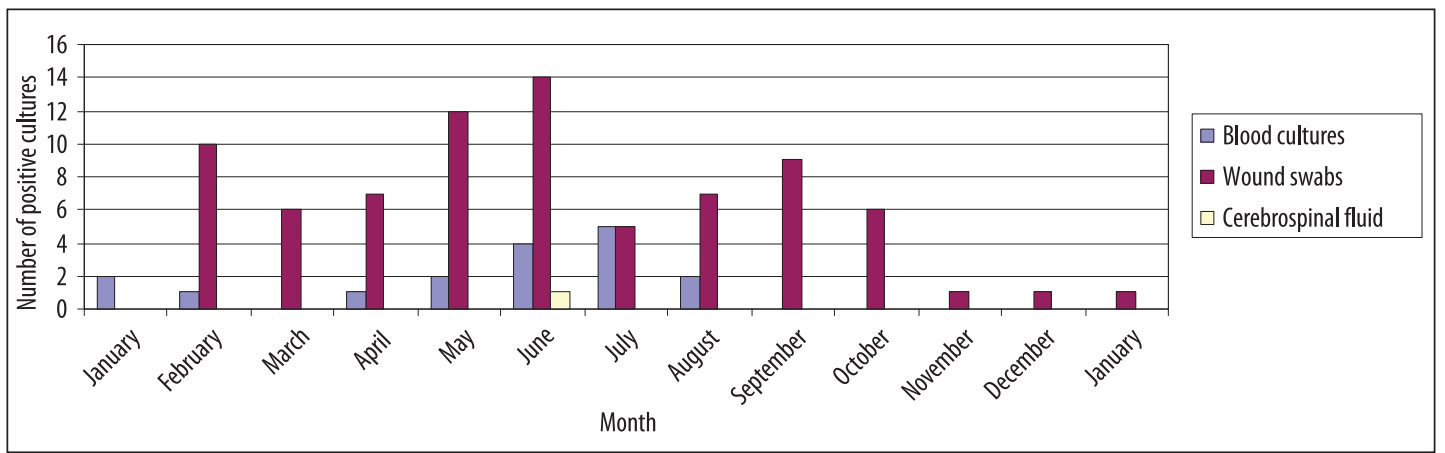

Figure 1 Laboratory-reported positive Serratia marcescens culture (cerebrospinal fluid, blood cultures, wound swabs) by month and culture site, January 2010 - January 2011.

and cerebrospinal fluid (1/96; 1\%).The highest number of positive cultures were registered in May and June $(33 / 96 ; 34.3 \%)$ (Figure 1 ).

The strains were identified with current phenotypic methods as Serratia marcescens with identical biochemical characteristics and antibiotic susceptibility patterns. The isolates showed resistance to ampicillin, amoxycillin-clavulanic acid, cefotaxime, ceftriaxon, ceftazidime, cefepime, trimethoprim/sulfometaxazole and gentamicin. Isolates remained susceptible to imipenem, meropenem, amikacin, ciprofloxacin, levofloxacin and piperacillin/tazobactam.

The infection control team was alerted and, after investigation, discovered the same phenotype of Serratia marcescens in a multiple-dose vial of anesthetic used during procedures.

All other environmental screens were negative for Serratia marcescens. The multiple-dose vial of anesthetic was changed with a separate vial in January 2011. Enhancement infection control measures were implemented and the outbreak was terminated in February 2011.

\section{Discussion}

Over the last 40 years, Serratia marcescens has become an important cause of nosoco- mial infections. There have been many reports concerning the identification, antibiotic susceptibility, pathogenicity and epidemiological investigation of this microorganism $(2,8)$. Outbreaks have been described in a variety of clinical settings and different environmental sources have been identified as reservoirs $(1,2,8)$. Accurate identification is important in defining outbreaks $(1,11)$.

In this study, we examined an outbreak of Serratia marcescens involving 79 patients, in the Orthopedic Clinic of the Clinical Center, University of Sarajevo. Before the onset of infections, all patients had been treated by orthopedic surgeons. Approximately within 48 hours after surgery the symptoms of infections occured. Laboratory findings showed elevated CRP, ESR, leukocytosis. Wound swabs, blood cultures and one cerebrospinal fluid sample from patients were sent to the Institute of Clinical Microbiology and all were positive for Serratia marcescens, with identical biochemical characteristics and antibiotic susceptibility patterns. All patients had episodes of clinically significant infection and required therapy with antibiotics. There were 17 episodes of septicemia, one meningitis and 78 wound infections. The situation was dramatic, and we decided to start an investigation. The Infection Control Team was alerted and an investigation carried out. Of the several pos- 
sible mechanisms for the pathogenesis of infections, given the nature of the outbreak (rapid onset post-surgery, complexity of the surgeries, severity of disease) a direct intravenous bolus of bacteria was suspected. This could have occurred if one or more of the following were contaminated: medications used during surgery, instruments, skin overlaying the site of surgery, or the hands of personnel performing the procedure (16, 17). Extensive environmental microbiological investigations were performed. A positive result for the epidemic strain of Serratia marcescens came from a multiple-dose vial of anesthetic used intravenously to provide general anesthesia during surgical procedures. All other environmental screens were negative for Serratia marcescens.

Both the epidemiologic and microbiologic evidence supported the contaminated vial of anesthetic as the cause of this outbreak. This is a very important finding, especially since in available literature we found very rare similar cases $(16,17)$. Halaby et al. (17) reported a case of fatal bacterial meningitis after spinal anesthesia. Several studies described infections caused by Serratia marcescens associated with contaminated disinfectants and antiseptics $(5,6,18-20)$. Sauter et al. (6) reported a case of meningitis associated with contamination of a skin antiseptic solution containing benzalkonium chloride. Bosi et al. (20) reported an outbreak of Serratia marcescens infection in the neurosurgery intensive care unit due to contamination of hexetidine solutions.

The outbreak in our hospital was extremely difficult to terminate, even with cohorting of patients, sterilization of equipment, reinforcement of hand-washing and deep-cleaning of facilities. As a result, enhancement infection control measures were implemented, as well as a recommendation for use of single vials. It was suggested that use of multi-dose vials is a constant threat, and separate vials should be used. Sepa- rate vials of medicines should be used for patients and the approach to procedures should be with all aseptic precautions.

Knowledge and practice of infection control measures are mandatory and should always be emphasized to staff. Continuous monitoring of nosocomial infections is essential. Nosocomial infections with multidrug-resistant organisms (MDROs) are a major cause of morbidity and mortality (1). A lot of patients were colonized with MDROs on admission, indicating the importance of screening on admission to intensive care units (ICUs). Screening on admission allows early detection and limits dissemination of these strains with application of appropriate control measures (21). The factors which predispose colonization and infection with MDROs are most often transmitted among patients by the hands of personnel. So, it was suggested that permanent control of personnel is necessary (21). The implementation of new control measures and replacement the multiple-dose vials of anesthetic with separate vials terminated the outbreak in February 2011.

We examined the outbreak using phenotypic methods. Phenotypic characterization of the isolates is useful for studying the relatedness of microbial pathogens. Relatedness of one clinical isolate to another during an outbreak is important in motivating the search for a common source or mode of transmission. Epidemiologically unrelated isolates could have similar characteristics and they may not be distinguished by phenotypic methods. Genotypic characterization of the isolates is necessary in these cases.

Unfortunately, in this study we did not characterize the examined isolates by using molecular methods because these methods have not been applied in our Institute. We know that it is limitation of this investigation, but we solved the problem using simple resources in the environment. Lately, molecular methods are being used more frequent- 
ly worldwide in typing hospital pathogens, since they allow genotypic characterization of the isolates (22). More recent studies with a wide range of strains and different primers have confirmed that RAPD-PCR is a promising method (22). Molecular methods have many advantages such as reproducibility, discriminatory power, ease of interpretation and performance. By applying this technique to all new isolates it should be possible to detect rapidly an outbreak of infection and allow the problem to be monitored and the source of the initial infection traced (22). This approach ensures a rapid and more adequate response by the infection control team, contributes to a reduction in the number of hospital infections and improvement of the quality of patient health care.

\section{Conclusion}

We examined the outbreak in our hospital using phenotypic methods. A positive result for the epidemic strain Serratia marcescens came from a multiple-dose vial of anesthetic used during procedures. Phenotypic characterization of the isolates is useful for studying the relatedness of microbial pathogens. Relatedness of one clinical isolate to another during an outbreak is important in motivating the search for a common source or mode of transmission. Phenotypic methods have some limitations, which can be overcome by using genotypic methods. Continuous monitoring of nosocomial infections is essential. Knowledge and practice of infection control measures is mandatory and should be always emphasized to staff.

Authors' contributions: Conception and design: MŠ; Acquisition, analysis and interpretation of data: MŠ; Drafting the article: MŠ; Revising it critically for important intellectual content: $\mathrm{MH}$.

Conflict of interest: The authors declare that they have no conflict of interest.

\section{References}

1. Emori TG, Gaynes RP. An overview of nosocomial infections, including the role of the microbiology laboratory. Clin Microbiol Rev. 1993;6(4):428-42.

2. Hejazi A, Falkiner FR. Serratia marcescens. J Med Microbiol. 1997;46:903-12.

3. Wheat RP, Zuckerman A, Rantz LA. Infection due to Chromobacteria: report of eleven cases. Arch Intern Med. 1951;88:461-6.

4. Dodson WH. Serratia marcescens septicaemia. Arch Intern Med. 1968;121:145-50.

5. Nakashima AK, Highsmith AK, Martone WJ. Survival of Serratia marcescens in benzalkonium chloride and in multiple-dose medication vials: relationship to epidemic septic arthritis. J Clin Microbiol. 1987;25:1019-21.

6. Sautter RL, Mattman KH, Legaspi RC. Serratia marcescens meningitis associated with a contaminated benzalkonium chloride solution. Infect Control. 1984;5:223-5.

7. Buffet-Bataillon S, Rabier V, Betremieux P, Beuchee A, Bauer M, Pladys P, et al. Outbreak of Serratia marcescens in a neonatal intensive care unit: contaminated unmedicated liquid soap and risk factors. J Hosp Infect. 2009;72(1):17-22.

8. Sleigh JD. Antibiotic resistance in Serratia marcescens. Br Med J. 1983;287:1651-3.

9. Palomar J, Puig M, Montilla R, Loren JG, Vinas M. Lipopolysaccharide recovery restores susceptibility levels towards beta-lactams in Serratia marcescens. Microbios. 1995;82(330):21-6.

10. Osano E, Arakawa Y, Wacharotayankun R, Ohta $\mathrm{M}$, Horii T, Ito $\mathrm{H}$, et al. Molecular characterization of an enterobacterial metallo beta-lactamase found in a clinical isolate of Serratia marcescens that shows imipenem resistance. Antimicrob Agents Chemother. 1994;38:71-8.

11. Gaynes R, Edwards JR; National Nosocomial Infections Surveillance System. Overview of nosocomial infections caused by gram-negative bacilli. Clin Infect Dis. 2005;41:848-54.

12. Bradford PA. Extended-spectrum beta-lactamases in the 21st century: characterization, epidemiology, and detection of this important resistance threat. Clin Microbiol Rev. 2001;14:933-51.

13. Jacoby GA, Munoz-Price LS. The new beta-lactamases. N Engl J Med. 2005;352:380-91.

14. Jorgansen HJ. Antibacterial agents and susceptibility test methods. In: Versolovic J, Caroll KC, Funke G, Jorgansen HJ, Landry ML, Warnock DW, editors. Manual of Clinical Microbiology. 
10th ed. (Vol 1). Texas: American Society for Microbiology; 2011.

15. Clinical and Laboratory Standards Institute. Performance Standards for Antimicrobial Susceptibility Testing. Fifteenth Informational Supplement. CLSI document M100-S15. Wayne, Pennsylvania: CLSI; 2005.

16. Schulz-Stubner S, Pottinger JM, Coffin SA, Herwaldt LA. Nosocomial infections and infection control in regional anaesthesia. Acta Anaesthesiol Scand. 2008;52(8):1144-57.

17. Halaby T, Leyssius A, Veneman T. Fatal bacterial meningitis after spinal anaesthesia. Scand J Infect Dis. 2007;39(3):280-3.

18. Knowles S, Herra C, Devitt E, O’Brien A, Mulvihill E, McCann SR, et al. An outbreak of multiply resistant Serratia marcescens: the importance of persistent carriage. Bone Marrow Transplant. 2000;25:873-7.

19. Arslan U, Erayman I, Kirdar S, Yuksekkaya S, Cimen O, Tuncer I, et al. Serratia marcescens sepsis outbreak in a neonatal intensive care unit. Pediatr Int. 2010;52(2):208-12.

20. Bosi C, Davin-Regli A, Charrel R, Rocca B, Monnet D, Bollet C. Serratia marcescens nosocomial outbreak due to contamination of hexetidine solution. J Hosp Infect. 1996;33:217-24.

21. Emily RM, Sydnor and Trish M. Hospital epidemiology and infection control in acute-care settings. Clin Microbiol Rev. 2011;24:141-73.

22. Hejazi A, Keane CT, Falkiner FR. The use of RAPD-PCR as a typing method for Serratia marcescens. J Med Microbiol. 1997;46:913-9. 